\title{
De-noising of Voltage Sag using Wavelet Transform
}

\author{
Suresh K. Gawre \\ Assist. Professor \\ Electrical Engg. Department \\ MANIT, Bhopal, India
}

\author{
N. P. Patidar \\ Associate Professor \\ Electrical Engg. Department \\ MANIT, Bhopal, India
}

\author{
R. K. Nema \\ Professor \\ Electrical Engg. Department \\ MANIT, Bhopal, India
}

\begin{abstract}
Wavelet analysis approach is presented in this paper for the automatic detection and analysis of voltage sag in power systems with noise and without noise. Wavelet analysis is used to process the voltage waveform for detection and better estimation of the time-related parameters of a voltage Sag in voltage supply, The method proposed, which can be processed in real-time, has been simulated for a low voltage distribution system, showing the potential in data reduction and accurate characterization of voltage Sag in power systems .
\end{abstract}

\section{Keywords}

Power quality, voltage Sag, wavelet analysis, De-noising.

\section{INTRODUCTION}

Huge demand of power electronics based apparatus and enhanced susceptibility of these devices causes the voltage disturbances. This paper reveals advantages of wavelet analysis for feature extraction of Sag (power quality disturbance) in small distribution system. Out of the different power quality events occurring in power systems, voltage Sags are probably one of the most important, because of their frequency of occurrence and the economical impact on commercial and industrial customers [2 and 11]. Symmetrical (for a three-phase or a three-phase-to-ground fault, asymmetrical (for a single-phase-to-ground or a two- phase or a two-phase-to -ground fault) or the sudden startup of motors are the common causes of voltage sag.

The development of new signal processing tools is required for on-line, real-time detection and analysis of voltage sag in order to avoid their consequences. As per definition sag has the wide range of different frequency bands, variation in magnitude and can be stationary or non-stationary which had been discussed [14]. The range power quality disturbances varies from low magnitude, low frequency $(0.1 \%$ and less than $25 \mathrm{~Hz}$ ) voltage fluctuation due welding and arc furnace type of intermittent loads to very high frequency transients $(0$ - 8.0pu, $5 \mathrm{MHz}$ ) caused by lightning strikes, switching and other related phenomena. Therefore identification and classification is not easy [9 and 21]. Also satisfy the Power Quality standards related to Sag is shown in table 1 and classification of power quality problem (SAG) is given in table 2 .
Table1: Power quality standards

\begin{tabular}{|c|l|l|l|}
\hline S.No. & Categories & Duration & $\begin{array}{l}\text { Voltage } \\
\text { magnitude }\end{array}$ \\
\hline \multicolumn{4}{|c|}{ Short duration variation } \\
\hline & \multicolumn{3}{|c|}{ SAG } \\
\hline a. & Instantaneous & $0.5-30$ cycle & $0.1-0.9 \mathrm{pu}$ \\
\hline b. & Momentary & 30 cycle -3 sec. & $0.1-0.9 \mathrm{pu}$ \\
\hline c. & Temporary & 3 sec. -1 min. & $0.1-0.9 \mathrm{pu}$ \\
\hline
\end{tabular}

Table 2: Classification of various power quality problem (SAG)

\begin{tabular}{|l|l|}
\hline Events & \multicolumn{1}{|c|}{ Standards } \\
\hline Classification of PQ & IEC 61000-2-5:1995, \\
& IEC1000-2-1:1990, \\
\hline Monitoring PQ & IEEE 1159:1995 \\
\hline Voltage sag/swell and & IEC 61009-2-1:1990, \\
interruptions & IEEE (493-1998, 1346- \\
& 1998) \\
\hline
\end{tabular}

Traditional methods presently being used by utilities for power quality monitoring are generally based on visual observation of voltage and current waveforms or conventional P Q indices like rms vale, THD, Power Factor etc.[14]. Highly automated monitoring hardware and software is required in order to provide sufficient coverage of complete system, to understand the prime causes of disturbances, to get better solutions and to predict future problems [1,6 and 24]. The large amount of PQ data deals with different practical problems in large storage and communication from local monitors to the major central processing unit. The organization of paper is such that, section- 2, provides basics of wavelet transform, followed by section- 3, explore the concept of basic data compression and proposed scheme, and section-4, deals with simulation and experimental result analysis.

\section{WAVELET TRANSFORM}

The wavelet transform is the projection of a discrete signal into two spaces: the approximation space and a series of detail spaces. The implementation of the projection operation is done by discrete-time sub band decomposition of input signals using filtering followed by down- sampling [4]. The wavelet transform is utilized to produce representative feature vectors that can accurately capture the characteristics of power quality disturbance, exploring feature extraction of disturbance signal to obtain dynamic parameters. 
Wavelet transform consists of a pair of transformations from one domain to another domain. The original domain is the time domain in wavelet transforms, while the transformed domain is called the time-scale domain. The transformation process from time domain to time-scale domain is a forward transform, because a given signal is decomposed into several other signals with different levels of resolution. It is possible to recover the original time domain signal without losing any information. This reverse process is called the inverse wavelet transform or signal reconstruction. These two processes compose the wavelet transform.

Let $x(t)$ be the time domain signal to be decomposed or analyzed. The dyadic wavelet transform (DWT) of $x(t)$ is then defined as

$$
D W T_{\Psi} x(m, n)=2^{-\frac{m}{2}} \int_{-\infty}^{\infty} x(t) \Psi^{*}\left(\frac{t-n 2^{m}}{2^{m}}\right) d t
$$

where $*$ denotes a complex conjugate, $m$ and $n$ are scale and time-shift parameters and $\boldsymbol{\Psi}(t)$ is a function of mother wavelet [5].

The DWT is implemented using a multiresolution pyramidal decomposition technique. A recorded time signal $c_{0}(n)$ with a sampled version of $x(t)$ is decomposed into its detailed $d_{l}(n)$ and smoothed $c_{l}(n)$ signals using filters $g(n)$ and $h(n) . g(n)$ has a band-pass filter response. Therefore, the filtered signal $d_{l}(n)$ is a detailed version of $c_{0}(n)$ and contains higher frequency components (such as sharp edges, transitions, and jumps in the original power disturbance signals) than the smoothed signal $c_{l}(n)$, because filter $h(n)$ has a low-pass frequency filter response. The decomposition of $c_{0}(n)$ into $c_{l}(n)$ and $d_{l}(n)$ is first-scale decomposition and they are defined as follows:

$$
\left\{\begin{array}{l}
c_{1}(n)=\sum_{k} h(k-2 n) c_{o}(k) \\
d_{1}(n)=\sum_{k} g(k-2 n) c_{o}(k)
\end{array}\right.
$$

Higher-order decompositions are performed in a similar manner [22].

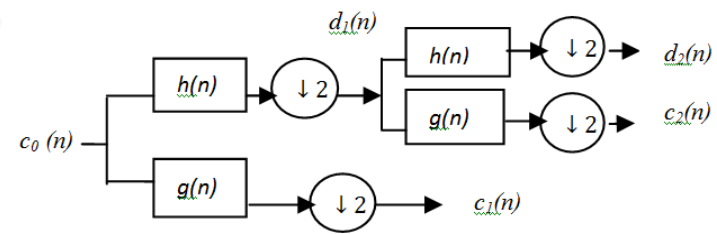

Fig 1: Decomposition of $c_{0}(n)$ into 2 scales

Because the family of the dilated wavelets constitutes an orthonormal basis for $L^{2}(R)$, the original signal $x(t)$ could be

potential as a new tool for automatically classifying power quality disturbances. Here important observations are:

- The reconstructed signal is actually a better signal for power quality analysis since it contains less electrical noise compared to the original signal [22].

- Wavelet transform analysis, commonly known as dyadic-orthonormal wavelet transform. Where the disturbance data decomposed into other signals which represent a smoothed version and a detailed recovered from its coefficients DWT $\boldsymbol{\Psi} x(m, n)$. The reconstructed signal is defined as:

$x(t)=2^{-\frac{m}{2}} \sum_{k} \sum_{n} D W T_{\Psi} x(m, n) \Psi^{*}\left(\frac{t-n 2^{m}}{2^{m}}\right)$

The magnitude of wavelet transform coefficients associated with PQ disturbance events is larger than normal signals. The compression is carried out in the wavelet domain by retaining wavelet transform coefficients associated with disturbance events and discarding all other disturbance free coefficients. The most-smoothed version of the original recorded signal $c_{0}(n)$ also kept for reconstruction purposes. Wavelet theory is an advanced mathematical tool that uses multiresolution techniques to analyze waveforms and images $[1,4,5,15]$. Wavelet analysis is capable of revealing aspects of data that other analysis tools would miss, including trends, breakdown points, discontinuities, and self-similarity. Feature extraction is a vital step that completes the link between intelligent analysis tools and actual PQ waveforms and data. Wavelet analysis has proven very strong and efficient in feature extraction from PQ disturbance data. Application of wavelet theory to PQ analysis has been well researched $[1,3,12,16,17$ and 25].

One very common application discussed and reviewed here is data compression as monitoring system deals with huge PQ Disturbance data collected in memory of data collection system. The large amounts of data pose several practical problems in the storage and communication of the data from local monitors to the central processing computers. Data compression has hence become paramount important issue in the PQ analysis area. Several methods using wavelet theory have been developed for the compression of PQ data [18][25].

\subsection{Wavelet Based Data Compression}

Wavelet transforms decompose, P Q signal into different scales at detailed resolutions. At each scale, the wavelet transform coefficients which associates with specific disturbance event are larger than those do not correspond to PQ disturbances, therefore specific disturbance coefficients are taking in account while others irrelevant events are leftover, so the amount of stored data can be drastically reduced. Now the compressed data can be used to reconstruct the original signal, with very little loss of information [20]. It was reviewed and discussed that the compression ratio, defined as the original file size divided by the compressed file size is well proved. These observation and discussions concludes that the Data storage requirement is minimized and transmission time while preserving the reconstructed signal in such a way that it is drastically reduced from the original [21]. Discussed approach found to be quite powerful in detecting and localizing various types of power quality disturbances. The basis of a possible disturbance classification scheme, using the squared wavelet transform coefficients, is also described. This study reveals new dimensions and offer great version of the original signal. The decomposition is performed using wavelet multi-resolution Analysis (MRA) by signal decomposition techniques [3-6].

○ Different compression is performed through signal decomposition, thresholding of wavelet transform coefficients, and signal reconstruction. 
After using the basic property of data compression, another application of WT as a tool of effective PQ detection is Denoising. De-noising of row signal is must because signals are always contaminated with noise which is directly measured using sensors and processed for monitoring purpose.

\subsection{De-noising of Sag via wavelet}

Noise reduction is considered as prime requirement in pre processing of data and data compression is necessary to reduce memory requirement of data storage system. Denoising is part of data compression several research work have been reported on wavelet based compression [9 - 24]. Generalized data compression scheme is shown in Fig. 5

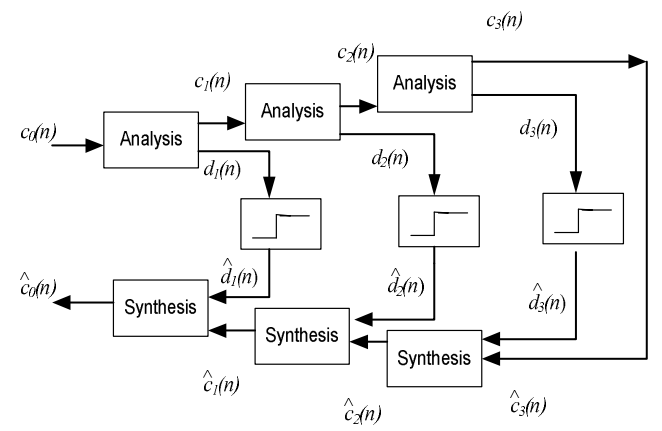

Fig 2: data compression scheme

To overcome the difficulties of extracting accurate features from PQ disturbance waveform in a highly noisy environment, a de-noising scheme is proposed as follows.

The idea discussed in [20] is that the magnitude of wavelet transform coefficients associated with PQ disturbance events is larger than normal signals. The compression is carried out in the wavelet domain by retaining wavelet transform coefficients (WTCs) associated with disturbance events and discarding all other disturbance free coefficients. The mostsmoothed version of the original recorded signal $c_{0}(n)$ also kept for reconstruction purposes. Thresholding of wavelet can be performed easily by discarding WTCs below a specific value which may vary scale to scale. Such threshold is based on the absolute maximum value of coefficients at associated signal details as given below in Eq. (4):

$H s=(1-\mu) \times \max \left\{\left|d_{s}(n)\right|\right\}$
Where $0 \leq \mu \leq 1$. For example, for $\mu=0.85$, the threshold THs is $15 \%$ of the absolute value of $d_{s}(n)$ therefore $\left|d_{s}(n)\right|$, that are smaller than THs are discarded, and those that are larger are stored. Now the signal is $\widehat{d_{s}}(n)$ in Eq.(5),

$$
\widehat{d_{s}}(n)= \begin{cases}d_{1}(n) & \left|d_{s}(n)\right| \geq T H s \\ 0 & \left|d_{s}(n)\right|<T H s\end{cases}
$$

Here the detailed WTCs given in Eq.(5) is used in reconstruction process and achieved approximate signal. Reduced noise and can be further data compression can be made easy, by choosing higher scales for MRA.

The large amounts of data pose several practical problems in the storage and communication of the data from local monitors to the central processing computers. Data compression has, hence become paramount important issue in the PQ analysis area. Several methods using wavelet theory have been developed for the compression of PQ data [1] [3] and [6].

\section{SIMULATION AND RESULTS}

The magnitude of fault-induced voltage dips at a certain point in the system depends mainly on the type and the resistance of the fault, the distance to the fault and the system configuration. The calculation of the dip magnitude for a fault somewhere within a radial distribution system requires the point of common coupling (PCC) between the fault and the load to be found. Figure 4. shows the voltage divider model,

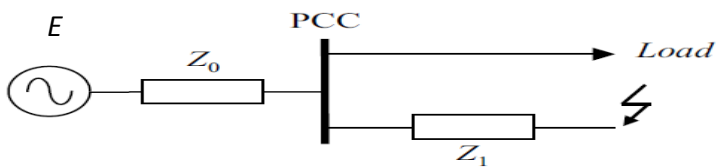

Fig 3: Voltage divider model for Sag

Where $Z_{0}$ is the source impedance, $Z_{1}$ is the impedance between the PCC and the fault (including any fault impedance). For three-phase faults only positive sequence impedances are needed. For single faults the sum of positive, negative and zero sequence impedances is needed. The dip magnitude $(\%)$ at the load position equals the voltage $(\%)$ at the PCC (if we neglect all load currents) 


\subsection{Test System for data generation}

A Small distribution System is simulated for generation of sag problem using MATLAB simulink (power system blockset)[15]. Sag problem has been created using Three phase fault block of simulink as shown in the Fig 4, and observed the results. Model parameters were selected as given in the Table 3.
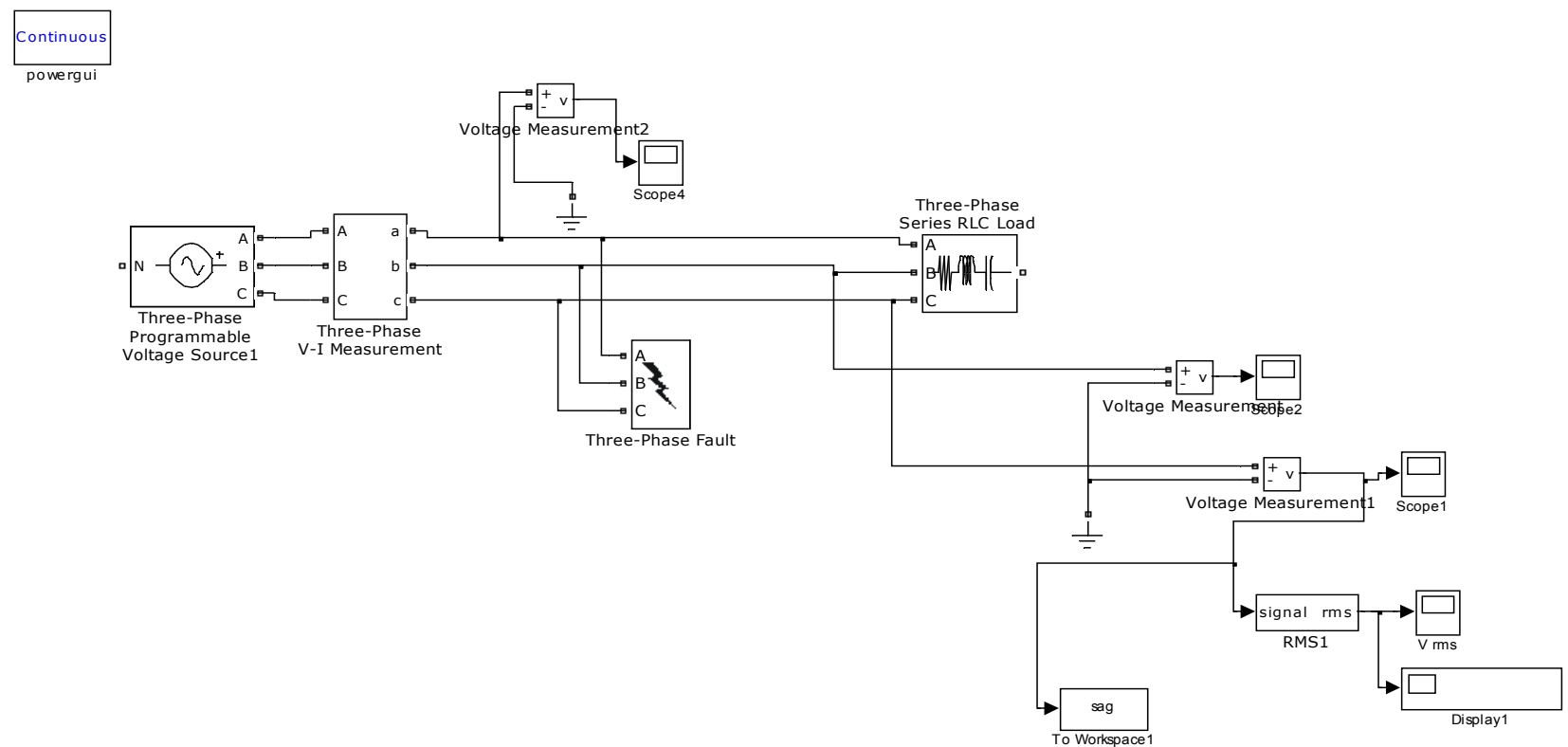

Table 3. Model Parameter

\begin{tabular}{|l|l|}
\hline Parameter & Value \\
\hline Source Voltage & $400 \mathrm{~V}(\mathrm{Ph}-\mathrm{Ph})$ \\
\hline Frequency & $50 \mathrm{~Hz}$ \\
\hline Fault resistance & $10 \Omega$ \\
\hline Ground resistance & $0.001 \Omega$ \\
\hline Snubbers resistance $\mathrm{Rp}$ & $1 \mathrm{e}^{6} \Omega$ \\
\hline Fault type & $\mathrm{LLG}$ \\
\hline
\end{tabular}

\section{Fig 4: MATLAB Simulation Model}

\subsection{Observations and Discussions.}

Simulation results have shown in the fig 5 . The four level decomposition of sag PQ disturbance which clearly detect the starting and ending point at detailed coefficient $\mathrm{d} 1$ and other details d2, d3, d4 are at different frequency bands. Here a4 is the approximate version of original signal, which provide noise free or smooth signal.

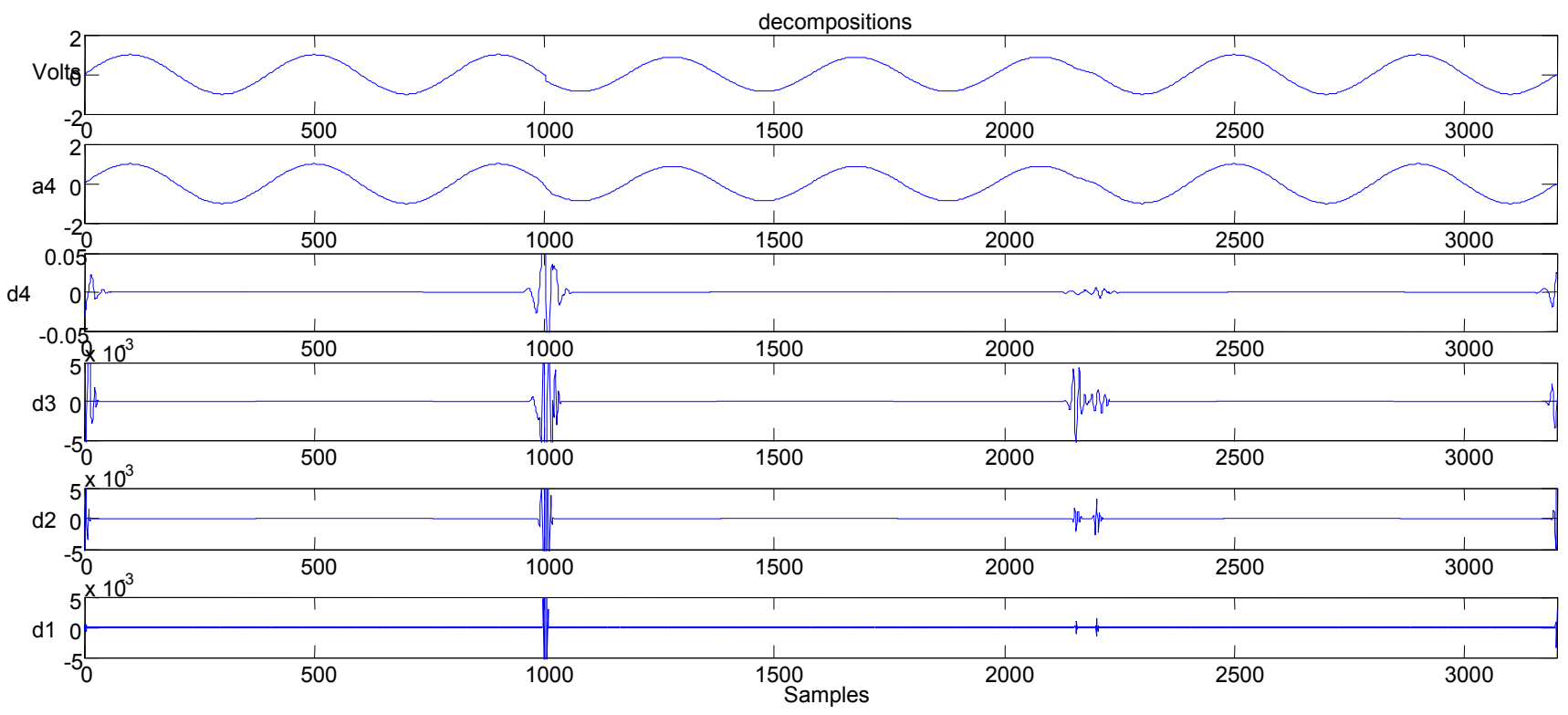

Fig 5 : MRA with four level decomposition 


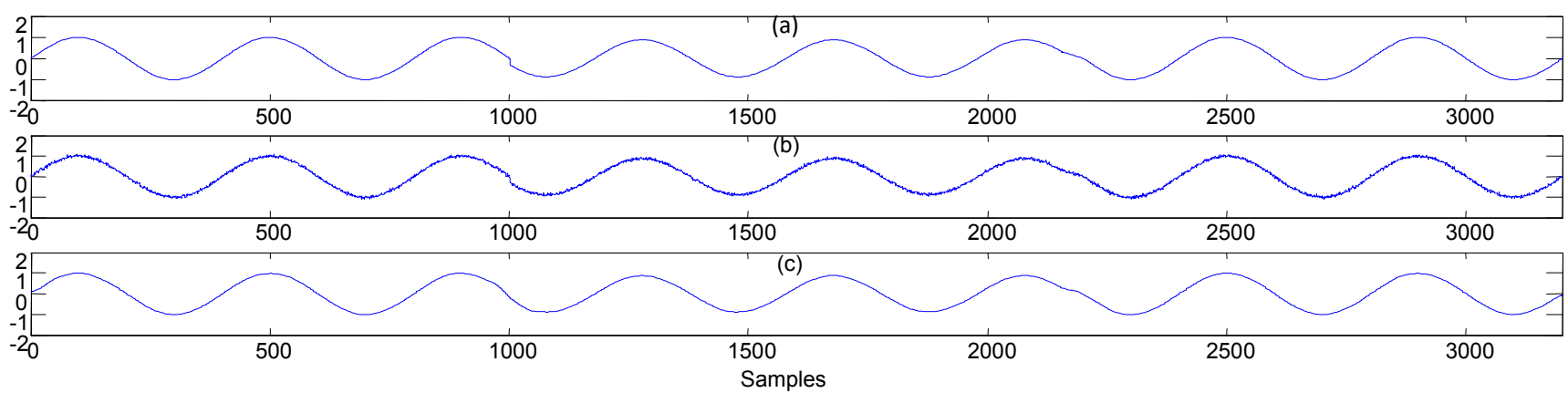

Fig 6: (a) Original signal, (b) Signal with noise and (c) De- noised signal

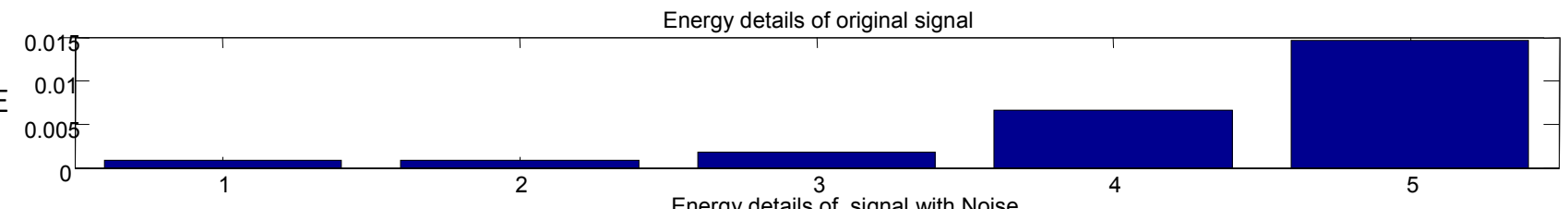
Energy details of signal with Noise
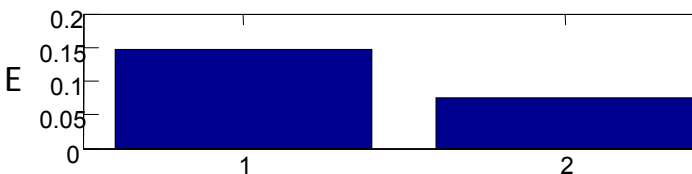

Energy details of de-noised signal
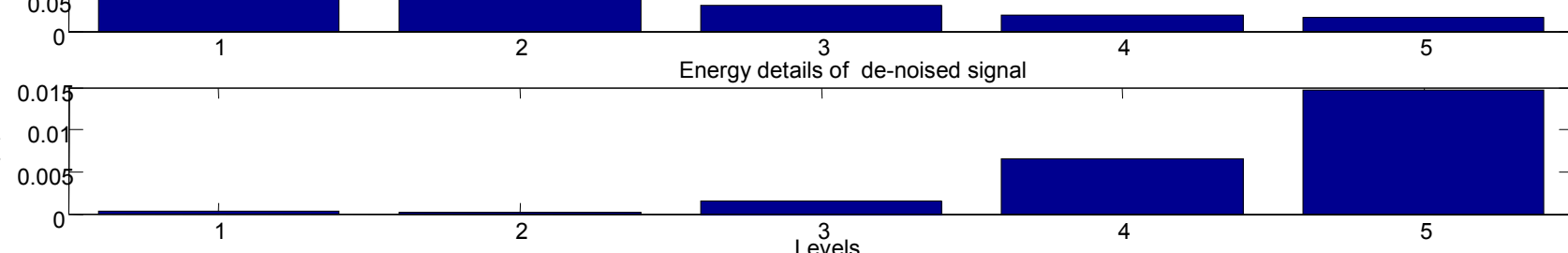

Fig 7: (a) Energy details of original, signal with noise and De-noised signal,

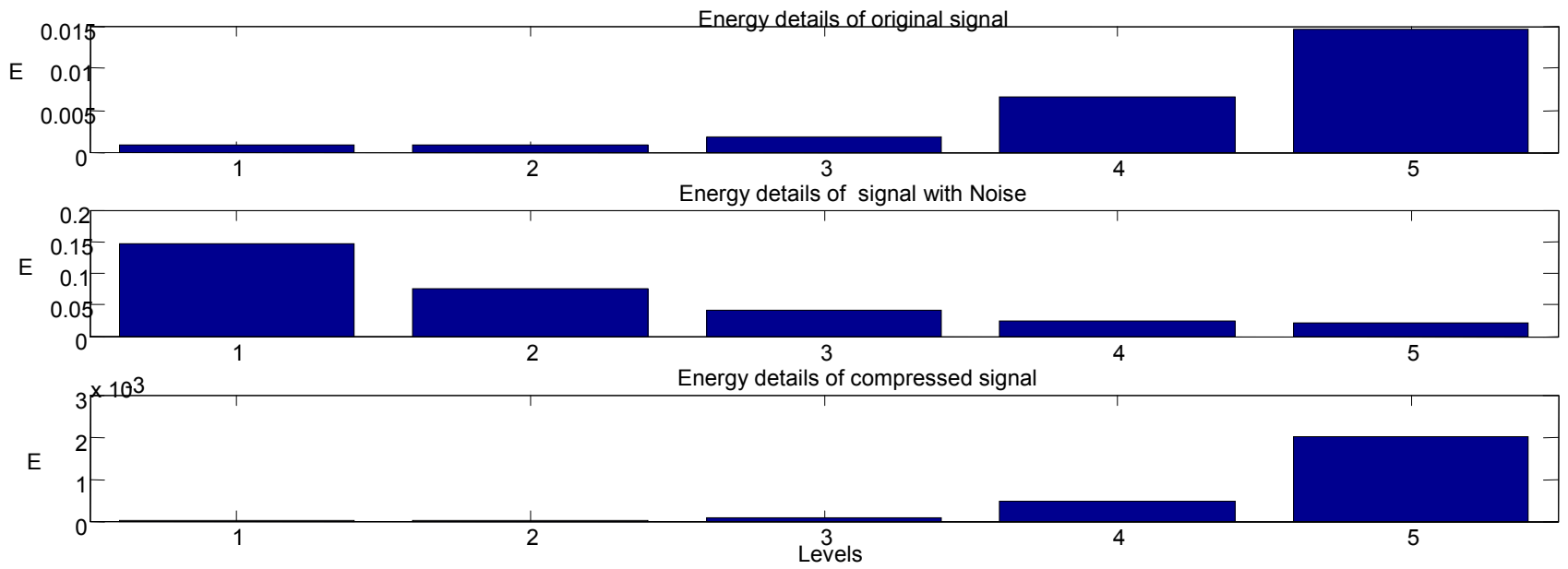

Fig 7: (b) Original, signal with noise and compressed signal

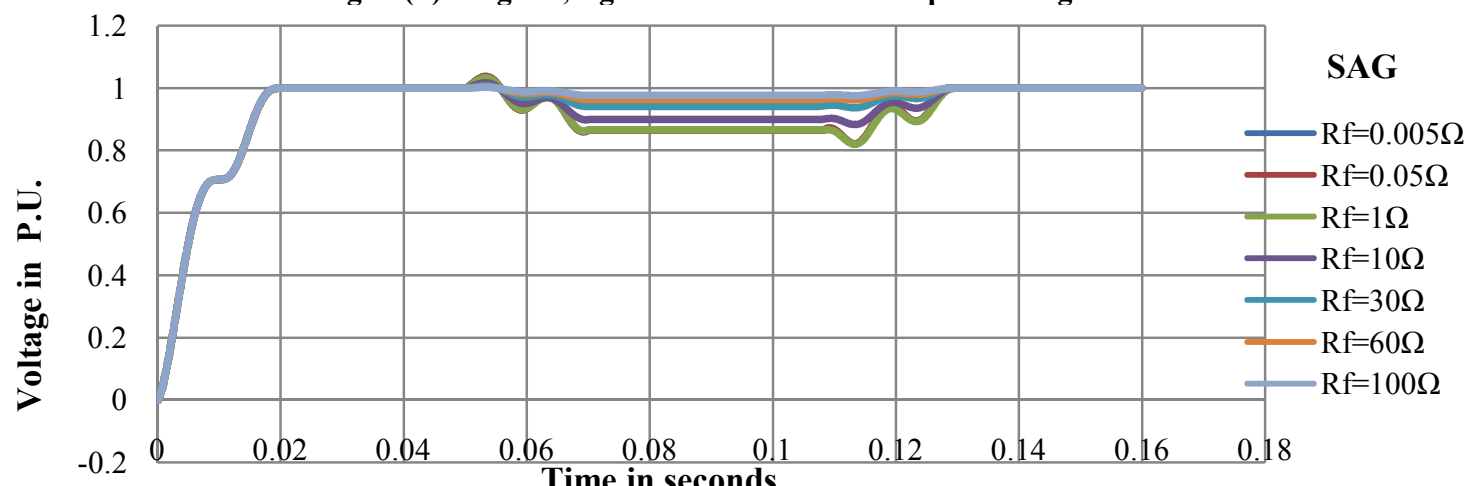

Fig 8: Variation of Voltage with fault resistance 
Program has been written in MATLAB to generate signal, add noise and then de- noising the signal using fixed threshold method and the results obtained are shown in Fig 6, where PQ signal original, with noise and De-noised signal is clearly observed.

The energy feature of wavelet coefficient at different details (from $\mathrm{d} 1$ to $\mathrm{d} 4$ ) as shown in Fig 7(a) and 7(b). The compressed data $(2-5 \%)$ can be used to reconstruct the original signal, with very little loss of information. Another observation remark that the Sag is deeper (Variation of voltage) with decrease of fault resistance as shown in Fig 8.

\section{CONCLUSIONS}

Power quality has become prime criteria in modern scenario of power system analysis. Approaches for automated detection, feature extraction and classification of $\mathrm{PQ}$ disturbances using wavelet transform, found excellent in the field of power quality monitoring and analysis. Further the memory space required for PQ data storage is drastically reduced.

Here results and observations drawn from the analysis, shows that the detection of PQ disturbance like SAG using wavelet transform gives better features. De-noising process employed here, provide robust method for feature extraction. Also the accuracy increases as noise is present while measuring the actual signal for power quality analysis which affects the actual signal. Further the wavelet transform can be added with AI Techniques for classification or real time monitoring of Power quality Disturbances.

\section{REFERENCES}

[1] Gaouda, A. M., Kanoun, S. H. Salama, M. M. A. and Chikhani, A. Y., "Pattern Recognition Applications for Power System Disturbance Classification" IEEE Transactions On Power Delivery, Vol. 17, No. 3, July 2002.

[2] Algira "Power Quality Following Deregulation" proceedings of the IEEE, vol. 88, no. 2, February 2000.

[3] Bousaleh G., Hassoun F., Ibrahim T. "Application of Wavelet Transform in the Field of Electromagnetic Compatibility and power quality of Industrial Systems" ACTEA 2009.

[4] Chui, C. K., An Introduction to Wavelets. New York: Academic, 1992.

[5] Sidney C., Burrus, Ramesh A. Gopinath and Haitao guo 'Introduction to wavelet and wavelet transform' Prentice Hall publication.

[6] El sayed Mohamed, Eldin Tag, "Characterization of power quality disturbances based on wavelet transforms" int. J. energy technology and policy, vol.4, nos1/ 2, 2006.

[7] Costa, F. B., Souza , B. A. and Brito, N. S. D., "RealTime Detection of Voltage Sags Based on Wavelet Transform" Transmission and Distribution Conference and Exposition: Latin America, 2010. IEEE/PES.

[8] IEEE power quality event characterization 1159.2, Feb. 2001.

[9] Liu, J. and Pillay, P., "Application of wavelet analysis in power system disturbance modeling," in Proc. 5th IEEE AFRICON Conf., Cape Town, South Africa, 1999, pp. 639-642.
[10] Jaehak Chung, Edward J. Powers, W. Mack Grady, and Siddharth C. Bhatt "An Automatic Voltage Sag Detector Using a Discrete Wavelet Transform and a CFAR Detector" IEEE, 2001.

[11] Chan, J. Y., Jovica V. Milanovic', and Alice Delahunty, "Risk-Based Assessment of Financial Losses Due to Voltage Sag” IEEE Transactions On Power Delivery, Vol. 26, No. 2, April 2011

[12] Agrisani, L., Daponte, P., Apuzzo, M. D., and Testa A., "A measurement method based on the wavelet transform for power quality analysis", IEEE Trans. Power Delivery, vol. 13, pp. 990-998, Aug. 1998.

[13] Bollen, M. H. J., "Understanding of power quality problems" IEEE Press.

[14] Bollen, M. H. J. and Gu, I. Y. H., Signal Processing of Power Quality Disturbances. Piscataway, NJ: IEEE Press, 2006.

[15] Misiti, M., Misiti, Y., Oppenheim, G., and Poggi, J. M., "Wavelet toolbox for use with Matlab-User's guide," The MathWorks, Natick,MA,1997.

[16] Poisson, O., Rioual, P., and Meunier, M., "New signal processing tools applied to power quality analysis," IEEE Trans. Power Delivery, vol.14, pp. 561-566, Apr. 1999.

[17] Pillay, P. and Bhattacharjee, A., "Application of wavelets to model short term power system disturbances," IEEE Trans. Power Syst., vol. 11, pp.2031-2037, July 1996.

[18] Bingham, R. P., Kreiss, D. G., and Santoso, S., "Advances in data reduction techniques for power quality instrumentation," in Proc. 3rd Eur.

[19] Santoso S., 'power quality assessment via wavelet transform analysis' IEEE transactions on power delevery, Vol 11, no.2, April 1996

[20] Santoso, S., 'power quality disturbance data compression using wavelet' IEEE transictions on power delevery, Vol 12, no.3, July 1997

[21] Littler, T. B. and Morrow, D. J., "Wavelets for the analysis and compression of power system disturbances," IEEE Trans. Power Delivery, vol. 14, pp. 358-364, Apr. 1999.

[22] Lachman, T., Memon, A. P., Mohamad, T. R. and Memon, Z. A., "Detection of Power Quality Disturbances Using Wavelet Transform Technique" International Journal For The Advancement Of Science \& Arts, Vol. 1, No. 1, 2010.

[23] Devaraju, T. et. al. "Role of custom devices in power Enhancement: A review" IJEST, Vol. 2(8), pp 36283634,2010

[24] yang W. R., "Modeling of wavelet based voltage sag monitoring system and design for the mixed signal integrated circuit implementation", 2008, IEEE.

[25] Gawre, Suresh K., Patidar, N..P. and Nema, R. K., "Application of wavelet transform in Power Quality: A Review", International Journal of Computer Applications (0975 - 8887) Volume 39- No.18, February 2012. 